\title{
EDUCAÇÃO E MOBILIDADE DIGITAL: DISCUTINDO E EXPERENCIANDO O USO DE TECNOLOGIAS MÓVEIS NA EDUCAÇÃO
}

Andreia Luiza Olivetto, Davi Fernando da Silva, Evandro Lombardi' Adriana Aparecida Lima Terçariol, Raquel Rosan Christino Gitahy

Universidade do Oeste Paulista - UNOESTE, Mestrado em Educação, Presidente Prudente, SP. E-mail: raquel@unoeste.br

\section{RESUMO}

$\mathrm{Na}$ atualidade, há uma disseminação do uso de aparelhos móveis na sociedade e no ambiente escolar. Neste cenário, a escola e os professores trabalham para conseguir lidar com estudantes cada vez mais conectados. Este trabalho teve por objetivo principal discutir sobre a aprendizagem e as tecnologias móveis no âmbito da formação continuada: a situação atual e as dificuldades enfrentadas pelos educadores, seja devido a paradigmas estabelecidos, falta de capacitação docente, escassez de conteúdo digital ou ainda, devido as políticas públicas com suas leis ultrapassadas ou inexistentes. Apresenta ainda as perspectivas futuras e recomendações para que, nos próximos anos, a educação desfrute plenamente desses recursos tecnológicos como forma de melhoria do processo ensino aprendizagem. Usou-se como metodologia uma revisão bibliográfica sistemática na base de dados Scielo e na Biblioteca Digital de Teses e Dissertações do IBICT por permitir o contato com produções acadêmicas científicas já publicadas, referentes ao tema pesquisado. A partir da pesquisa, pode-se traçar um panorama da situação atual, as dificuldades para a utilização das tecnologias móveis, o que tem sido realizado e as perspectivas para o futuro da aprendizagem móvel.

Palavras-chave: aprendizagem, TIC, políticas educacionais, tecnologia móvel

\section{EDUCATION AND DIGITAL MOBILITY: ARGUING AND EXPERIMENTING THE USE OF MOBILE TECHNOLOGY ON EDUCATION}

\begin{abstract}
Currently, there is a widespread use of mobile devices in society and at school environment. In this scenario, the school and the teachers work to handle with students increasingly connected. This study had the main objective to discuss about learning and mobile technologies in the context of continued education: the current situation, the difficulties faced by educators, whether due to established paradigms, lack of teacher training, shortage of digital content or because the public policies with their outdated or nonexistent laws. It also presents future perspectives and recommendations so, in the next years, education can be able to fully enjoy these technological resources as a way to improve the learning process. It was used as methodology a systematic literature review on the Scielo database and Digital Theses and Dissertations IBICT Library for allowing contact with scientific academic productions already published on the topic searched. From the research, we can draw a picture of the current situation, the difficulties in the use of mobile technologies, what has been done and the prospects for the future of mobile learning.
\end{abstract}

Keywords: learning, TIC, educational policies, mobile technology. 
INTRODUÇÃO

A mobilidade é um dos principais fenômenos tecnológicos. Ela permite ao usuário desenvolver atividades que envolvam o acesso à internet em qualquer lugar e esta tecnologia está sendo incorporada aos espaços e práticas escolares, num movimento que vincula a escola à cultura digital.

Aprendizagem móvel refere-se ao uso das tecnologias móveis, smartphones, notebooks, tablets e outros, isoladas ou em combinação com outras práticas metodológicas educacionais, a fim de permitir uma aprendizagem contínua e significativa, em qualquer lugar. Este artigo se originou da experiência vivida pelos pesquisadores, autores deste estudo, na elaboração e apresentação de um seminário intitulado "Tecnologia Móvel e Educação", na disciplina "Práticas Docentes e Novas Tecnologias", vinculada ao Programa de Pós-Graduação em Educação ((Mestrado em Educação) da Universidade do Oeste Paulista (UNOESTE/Presidente Prudente/SP).

Construído neste contexto, o artigo tem como objetivo partilhar a experiência vivida na elaboração e apresentação desse seminário, bem como refletir sobre a aplicabilidade das tecnologias móveis em contextos educacionais na atualidade e discutir as perspectivas futuras, os desafios e a quebra de paradigmas para uma melhor inserção de recursos móveis na educação como um todo.

A seguir é apresentada a metodologia adotada para o desencadeamento e a organização deste estudo, seguida da apresentação dos resultados e breve discussão, e por fim, algumas considerações.

\section{METODOLOGIA}

A construção deste artigo iniciou-se com a realização de um levantamento bibliográfico em diferentes fontes, dentre elas, destaca-se a base de dados SciELO. Para esse levantamento foram utilizados os seguintes descritores: tecnologias móveis na educação, uso do celular, notebook e tablets em sala de aula, processo de avaliação on-line, mobile learning, entre outros.

Após a busca, sistematização e análise destes trabalhos encontrados nessa base de dados, foi elaborada uma apresentação de slides com informações sintetizadas sobre a temática abordada, com o intuito de utilizarmos esse material como roteiro no desenvolvimento do seminário a ser apresentado aos demais pós-graduandos participantes da disciplina "Práticas Docentes e Novas Tecnologias", conforme já mencionado. 
Ao fim da apresentação, foi aplicado teste on-line, utilizando o aplicativo Socrative ${ }^{1}$, uma ferramenta gratuita, multi-plataforma, que possibilita interatividade entre os alunos, mas, principalmente a avaliação do entendimento do conteúdo abordado no seminário, de forma dinâmica, criativa e lúdica. Esse teste consistiu na aplicação de perguntas (previamente preparadas pelos membros do grupo sobre o conteúdo abordado no seminário), utilizando a plataforma Socrative Teacher).

A partir dessas perguntas, foram apresentadas aos participantes opções de respostas de múltipla escolha, na plataforma Socrative Student (interface do estudante). Nas imagens abaixo vemos a página inicial do Socrative (teacher) e exemplo de questão aplicada:

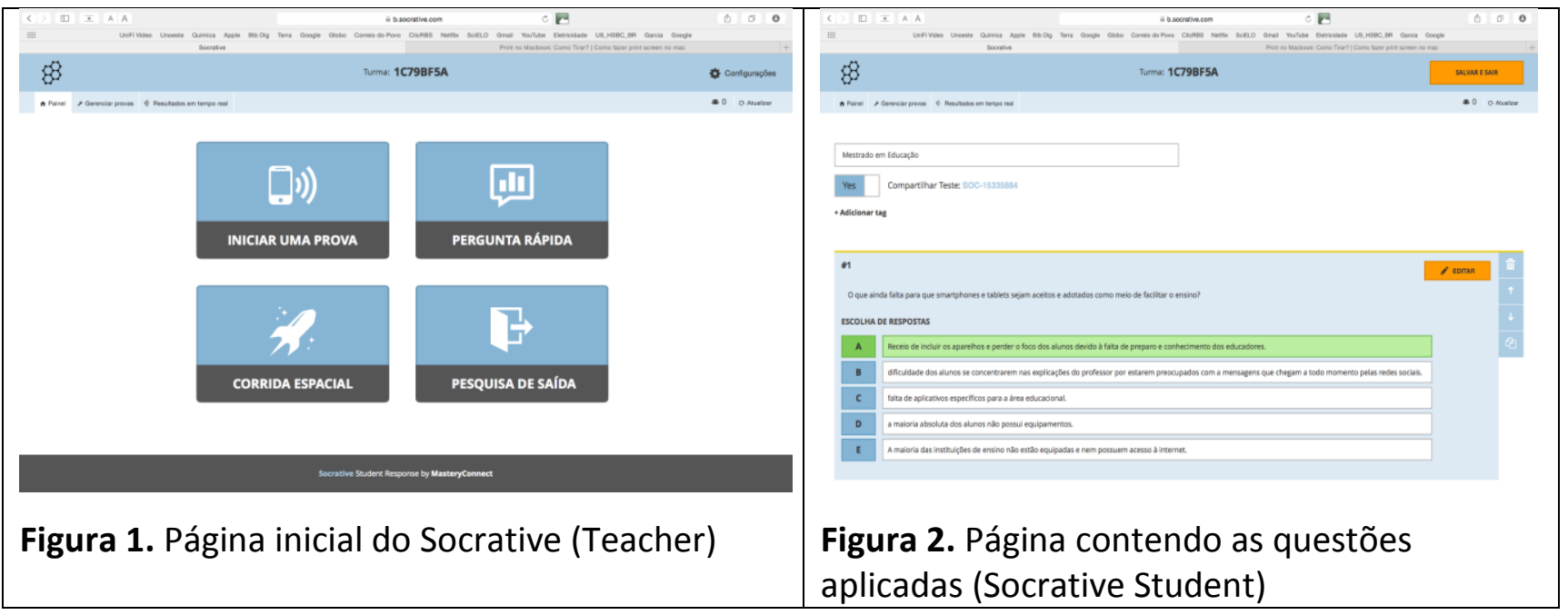

Ao final, a plataforma mostrou o percentual de acertos entre os participantes e possibilitou a plena visualização da interatividade, a partir do número de erros e acertos. Na aplicação desse teste os participantes utilizaram recursos computacionais móveis como notebook, smartphones, tablets, e afins, com acesso a internet no ambiente da sala de aula.

\footnotetext{
${ }^{1}$ Socrative BymasteryConnect, disponivel em: http://www.socrative.com/ 


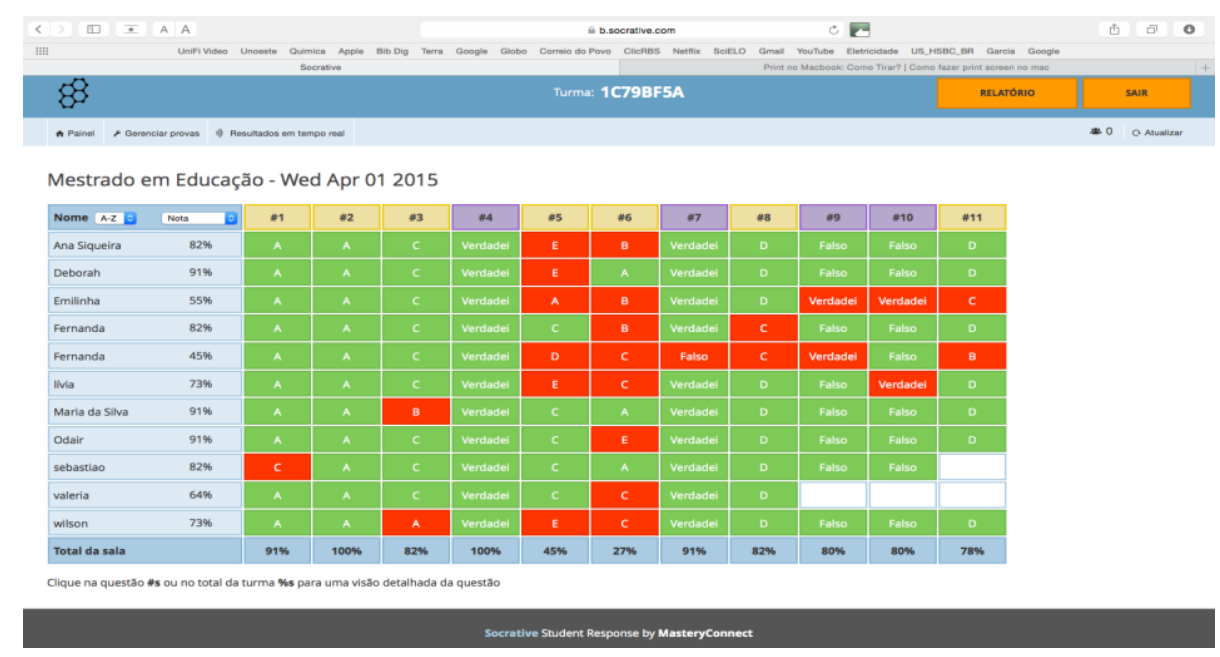

Figura 3. Tabela contendo as respostas dos alunos e o porcentual de acertos. O professor acompanha em tempo real.

Dentre as tecnologias móveis utilizadas nessa dinâmica, optamos por focar a análise no smartphone, por ser um dispositivo presente no cotidiano de muitos estudantes e pela sua portabilidade. A seguir apresentamos e discutimos os principais resultados alcançados com essa experiência.

\section{RESULTADOS E DISCUSSÃO}

$\mathrm{Na}$ apresentação do seminário, foi possível verificar que entre os pós- graduandos há muito interesse na utilização das tecnologias móveis e que os motivos e recomendações para sua utilização são perfeitamente coerentes e aplicáveis ao universo educacional, no qual estão inseridos.

A dinâmica realizada com o aplicativo Socrative deu a todos uma nova opção para aplicação de testes e avaliações, possibilitando imediata verificação da assimilação dos conteúdos, podendo ser utilizado desde o nível fundamental até o superior, porém com maior aplicabilidade, a partir do nível médio, tanto em escolas públicas como privadas.

Foi relatado pelos pós-graduandos, que após o seminário, utilizaram a tecnologia móvel em suas salas de aula, com a aplicação de testes, a partir do aplicativo Socrative, obtendo sucesso, o que os motivou a comentar que adotariam o mesmo como método alternativo de avaliação.

Atualmente, temos visto que existe uma grande resistência por parte dos docentes e administradores escolares em permitir ou utilizar as tecnologias móveis na educação. Isso ocorre devido a alguns fatores, dentre eles podemos citar: a proibição da utilização de celulares nas escolas da rede pública no estado de São Paulo, conforme Decreto no. 52.625, de 15 de janeiro de 
2008; o desconhecimento da tecnologia e dos aplicativos educacionais por parte dos educadores; a falta de políticas educacionais para o uso de tecnologias móveis; o currículo escolar não aborda adequadamente o uso das novas tecnologias; a escola e/ou alunos não possuem os recursos tecnológicos; receios quanto a exposição do cotidiano da escola, devido a possibilidade de elaboração, por parte dos alunos, de vídeos e fotos inadequados.

Durante a apresentação do seminário, aberta a discussão com os educadores/pósgraduandos, foi possível confirmar a veracidade destes problemas, em função dos relatos das experiências profissionais dos mesmos.

De acordo com as pesquisas realizadas na elaboração do trabalho, identificam-se algumas iniciativas de sucesso, que veem sendo realizadas atualmente em escolas do Brasil e do mundo, nas quais se destacam, na educação formal os "programas UCA": Um computador por aluno; as iniciativas "traga seu próprio dispositivo - BYOD": utilizando os recursos tecnológicos dos alunos para acessar conteúdos educacionais; a "sala de aula invertida": onde os alunos são estimulados a realizar pesquisas sobre determinado assunto e trazer para a sala de aula suas pesquisas e duvidas para complementação e esclarecimentos do professor; a utilização de Gadgets: aparelhos como o "Clickers" e softwares como "Socrative" para enquetes e avaliações em sala de aula ou fora dela; e os Ambientes Virtuais de Aprendizagem (AVA): plataformas de Educação a Distância (EaD).

Já no contexto da educação informal, contexto onde a aprendizagem móvel tem se desenvolvido mais facilmente, temos como destaques: os livros digitais (didáticos ou não); aplicativos móveis educacionais; ferramentas de busca e pesquisa, que permitem estender o processo de aprendizagem para todos os ambientes e em qualquer horário, o que é comumente chamado de aprendizagem contínua (Seamless).

Diversos são os motivos que incentivam a utilização das tecnologias móveis na educação, entre eles podemos destacar as facilidades que possibilitam a aprendizagem em qualquer hora e lugar, quebrando barreiras entre o aprendizado formal e o informal; a otimização do tempo em sala de aula que possibilita aos educadores um tempo adicional com os alunos para aprofundar e fixar os conteúdos; aplicativos educacionais com recursos variados oferecem aos educadores oportunidades de personalizar o aprendizado; os fóruns virtuais e a interação dos jogos e aplicativos educativos permite o desenvolvimento de grupos de estudo e comunidades virtuais de alunos e educadores; aplicativos de avaliação, como o Socrative, proporcionam formas de avaliação e feedback imediatos, o que traz agilidade para verificar os resultados dos métodos utilizados e o aproveitamento dos alunos; as tecnologias móveis possibilitam também a inclusão social de alunos com deficiência e os auxilia no aprendizado, facilitando a comunicação e assim 
impulsionando o aprendizado e a interação educacional dos portadores de deficiência visual e auditiva.

Considerando que grande parte dos alunos, principalmente do ensino fundamental e médio, já nasceram em contato com as tecnologias móveis, e que a organização do conhecimento nesta geração é facilitada pelas formas de comunicação que são utilizadas nos aplicativos, softwares e jogos eletrônicos, instigamos aos educadores/pós-graduandos a pensar em: Como as escolas devem lidar com esta realidade?

Esta discussão no seminário nos levou a afirmar que as escolas devem buscar a personalização do aprendizado e proporcionar a aprendizagem contínua, não sendo mais aceitável distanciar-se da realidade dos alunos, para tanto faz-se necessário revisar as politicas educacionais para inserir as tecnologias móveis no contexto formal da educação.

Por ter se popularizado tanto, estima-se que, no final de 2012, o número de dispositivos móveis ultrapassou o total da população mundial (CISCO, 2012). Um excelente motivo para a utilização desta tecnologia a favor da educação.

Apesar do progresso alcançado nas últimas duas décadas ser bastante significativo, ainda há muito por fazer para que os frutos de uma aprendizagem móvel sejam plenamente significativos. A grande maioria das políticas de TIC antecedem o advento dos aparelhos móveis e as raras políticas existentes, ou os tratam tangencialmente ou proíbem o seu uso em sala de aula. Outro grande desafio consiste em fornecer apoio, capacitação, acesso e formação para os docentes além de incentivar a criação de conteúdo digital para esses aparelhos móveis e ampliar e melhorar as opções de conectividade. Por fim, desenvolver programas e campanhas assegurando a equidade e o acesso a todos os cidadãos além de promover o uso seguro, responsável e saudável das tecnologias móveis.

Segundo relatório fornecido pela UNESCO (2014) apesar do grande número de assinaturas de telefone celular no mundo, muitos professores, formuladores e educadores ainda não usufruem ao máximo a capacidade deste aparelho no âmbito educacional. Nos próximos anos, a tecnologia dará um salto nunca antes visto. A tecnologia será mais barata, acessível e funcional; os dispositivos móveis conseguirão coletar, armazenar e sintetizar mais dados do que conseguem fazer atualmente, o que possibilitará uma revolução no modo de ensinar e de aprender; a barreira da linguagem será superada; as fontes de energia dos aparelhos serão mais duradoras.

Com estas melhorias, o futuro da aprendizagem móvel se revela promissor e imensamente recomendável para o avanço da sua empregabilidade nas metodologias de ensino. 


\section{CONSIDERAÇÕES FINAIS}

Há uma diferença crucial na utilização das tecnologias móveis nas escolas brasileiras atualmente. No ensino fundamental e médio pode-se notar claramente que nas escolas públicas, em função da carência dos alunos, da falta de incentivos, do despreparo dos educadores e administradores, as tecnologias móveis não estão disponíveis ou são subutilizadas. Enquanto que nas escolas particulares, ocorre o contrário, os alunos possuem celulares e tablets, os educadores e os pedagogos são estimulados a buscar novas formas para propiciar a construção do conhecimento, pressionados, entre outras coisas, pela concorrência entre as escolas. No ensino superior, a distância entre os universos: público e privado é menos perceptível, a grande maioria dos alunos possui dispositivos móveis e as universidades têm disponibilizado recursos e métodos para sua utilização como, por exemplo, EaD e fóruns virtuais.

Tendo em vista que, grande parte da população tem acesso às tecnologias móveis e sendo impossível ignorar a presença destas e sua influência na forma de vida das pessoas na atualidade, concluímos ao término deste estudo que é fundamental que as escolas cedam espaço à utilização das tecnologias móveis como forma de aproximar a escola da realidade dos alunos, aproveitando todos os benefícios descritos acima.

Para que isso ocorra, há várias recomendações incluindo a criação ou alteração de políticas educacionais que possibilitem a disseminação das tecnologias móveis na educação. No entanto, para que as novas diretrizes sejam aplicadas, faz-se necessário conscientizar os estudantes, os educadores e demais membros das instituições educacionais sobre sua importância e sobre os benefícios que podem ser alcançados com sua aplicação.

Os investimentos governamentais na expansão e melhoria das opções de conexão são extremamente necessários para que as metodologias de ensino, baseadas na utilização de tecnologias móveis, sejam utilizadas por todos os seguimentos da comunidade escolar.

Adicionalmente é necessário ampliar e otimizar o desenvolvimento de conteúdos educacionais acessáveis em aplicativos móveis. Uma vez que, os conteúdos educacionais devem estar disponíveis em diferentes formatos e acessíveis em distintos ambientes, os educadores terão assim oportunidade de aproveitar estes meios para propiciar aos estudantes, ritmo e formato mais adequado e produtivo para que desenvolvam suas habilidades, personalizando a aprendizagem.

Enfim, para que o futuro da aprendizagem móvel seja uma realidade nas escolas, na vida dos estudantes e da população em geral, faz se necessário que sejam estabelecidas medidas governamentais que, entre outras ações, possibilitem o acesso à internet, forneçam estrutura e 
formação aos educadores e gestores das instituições de ensino, adequem os currículos escolares, e coloquem em prática políticas educacionais que proporcionem a utilização das facilidades que as tecnologias móveis proporcionam.

\section{REFERÊNCIAS}

CISCO http://www.cisco.com/web/PT/press/articles/2012/20120601.html.

http://www.al.sp.gov.br/repositorio/legislacao/decreto/2008/decreto-52625-15.01.2008.html.

PÉREZ DE PABLOS, Susana - Sete Motivos para Ligar o Celular em Sala de Aula - Disponível em: http://educacao.uol.com.br/noticias/2015/02/24/sete-motivos-para-ligar-o-celular-na-sala-deaula.htm - Acessado em 13-03-2015.

PORVIR - O futuro se aprende - Aprendizagem Móvel - Disponível em:

http://porvir.org/wiki/aprendizagem-movel.

UNESCO, 2014, Diretrizes de Políticas da UNESCO para Aprendizagem Móvel - Disponível em: www.unesco.org/open-access/terms-use-ccbyncnd-port - Acessado em 14/03/2015.

UNESCO, 2014, O Futuro da Aprendizagem Móvel, Implicações para planejadores e gestores de políticas - Disponível em: www.unesco.org/open-access/terms-use-ccbyncnd-port - Acessado em $14 / 03 / 2015$.

YAMAMOTO, Karina - UOL, em São Paulo - 23/01/2015 - Por que a tecnologia não mudou a educação: porque o sistema é o mesmo - Disponível em:

http://educacao.uol.com.br/noticias/2015/01/23/por-que-a-tecnologia-nao-mudou-a-educacaoporque-o-sistema-e-o-mesmo.htm - Acessado em: 13-03-2015 